\title{
Targeted PET Imaging of Chemokine Receptor 2-Positive Monocytes and Macrophages in the Injured Heart
}

\author{
Gyu Seong Heo*1, Geetika Bajpai*2, Wenjun Li*3, Hannah P. Luehmann ${ }^{1}$, Deborah H. Sultan ${ }^{1}$, Hao Dun ${ }^{2}$, \\ Florian Leuschner $^{4}$, Steven L. Brody ${ }^{1,2}$, Robert J. Gropler ${ }^{1}$, Daniel Kreisel ${ }^{3,4,5}$, Kory J. Lavine ${ }^{2,5,6}$, and Yongjian Liu ${ }^{1}$ \\ ${ }^{1}$ Department of Radiology, Washington University School of Medicine, St. Louis, Missouri; ${ }^{2}$ Department of Medicine, Washington \\ University School of Medicine, St. Louis, Missouri; ${ }^{3}$ Department of Surgery, Washington University School of Medicine, St. Louis, \\ Missouri; ${ }^{4}$ Department of Internal Medicine III, University of Heidelberg, Heidelberg, Germany; ${ }^{5}$ Department of Immunology and \\ Pathology, Washington University School of Medicine, St. Louis, Missouri; and ${ }^{6}$ Department of Developmental Biology, Washington \\ University School of Medicine, St. Louis, Missouri
}

Proinflammatory macrophages are important mediators of inflammation after myocardial infarction and of allograft injury after heart transplantation. The aim of this study was to image the recruitment of proinflammatory chemokine receptor 2-positive $(\mathrm{CCR} 2+)$ cells in multiple heart injury models. Methods: ${ }^{64} \mathrm{Cu}$-DOTA-extracellular loop 1 inverso (ECL1i) PET was used to image CCR2+ monocytes and macrophages in a heart transplantation mouse model. Flow cytometry was performed to characterize CCR2+ cells. Autoradiography on a human heart specimen was conducted to confirm binding specificity. ${ }^{64} \mathrm{Cu}$ - and ${ }^{68} \mathrm{Ga}-\mathrm{DOTA}-\mathrm{ECL} 1 \mathrm{i}$ were compared in an ischemia-reperfusion injury mouse model. Results: ${ }^{64} \mathrm{Cu}-$ DOTA-ECL1i showed sensitive and specific detection of CCR2+ cells in all tested mouse models, with efficacy comparable to that of ${ }^{8} \mathrm{Ga}$-DOTA-ECL1i. Flow cytometry demonstrated specific expression of CCR2 on monocytes and macrophages. The tracer binds to human CCR2. Conclusion: This work establishes the utility of ${ }^{64} \mathrm{Cu}-\mathrm{DOTA}-\mathrm{ECL} 1 \mathrm{i}$ to image CCR2+ monocytes and macrophages in mouse models and provides the requisite preclinical information to translate the targeted clinical-grade CCR2 imaging probe for clinical investigation of heart diseases.

Key Words: PET; C-C chemokine receptor type 2 (CCR2); monocytes; macrophages; heart injury

J Nucl Med 2021; 62:111-114

DOI: 10.2967/jnumed.120.244673

$\mathbf{W}_{\mathrm{i}}$

thin the heart, macrophages represent the most abundant immune cell type and have important roles in potentiating both inflammatory and reparative responses after cardiac injury. Recent studies have shown that chemokine receptor 2-positive $(\mathrm{CCR} 2+)$ monocytes and macrophages, recruited to the site of myocardial injury, are important mediators of adverse remodeling in myocardial infarction (MI), heart failure pathogenesis, and inflammation after heart transplantation. Thus, CCR2 + cells represent a potential

Received Mar. 6, 2020; revision accepted Apr. 16, 2020.

For correspondence or reprints contact: Daniel Kreisel, Kory J. Lavine, or Yongjian Liu, Department of Radiology, 510 S. Kingshighway Blvd., Campus Box 8225, St. Louis, MO 63110.

E-mail: kreiseld@wustl.edu, klavine@wustl.edu, or yongjianliu@wustl.edu

${ }^{*}$ Contributed equally to this work.

Published online May 22, 2020.

COPYRIGHT @ 2021 by the Society of Nuclear Medicine and Molecular Imaging. therapeutic target to improve outcomes in the above disease settings (1-3).

Though some PET tracers have been developed for MI imaging (4-6), these probes lack specificity in visualizing cell types important to the remodeling process. Molecular imaging of CCR2+ monocytes and macrophages provides not only a sensitive approach to determine a specific mechanism of myocardial inflammation that is therapeutically targetable but also a strategy to facilitate the selective delivery of immunomodulatory agents to cardiovascular patients who are most likely to benefit.

Previously, we reported the capacity of a targeted PET tracer to quantify the CCR2-specific inflammation burden in multiple animal models using extracellular loop 1 inverso (ECL1i) peptide through ${ }^{64} \mathrm{Cu}$ or ${ }^{68} \mathrm{Ga}$ radiolabel (7-9). The PET signal intensity in inflammatory tissues was corroborated by molecular characterization of CCR2 expression and correlated with disease progression and regression. Currently, ${ }^{64} \mathrm{Cu}$ radiolabeled ECL1i $\left({ }^{64} \mathrm{Cu}\right.$-DOTA-ECL1i) is approved by the Food and Drug Administration for clinical investigations to identify CCR2 + cells in humans (10). To explore its potential for imaging patients with heart injury, we assessed the sensitivity and specificity of ${ }^{64} \mathrm{Cu}-$ DOTA-ECL1i CCR2 + monocyte and macrophage abundance in preclinical models of heart transplantation and MI and in comparison with ${ }^{68} \mathrm{Ga}$ radiolabeled analog. These studies provided strong support to apply the CCR2 PET imaging strategy to human subjects.

\section{MATERIALS AND METHODS}

\section{Mouse Heart Injury Models}

For the heart transplantation mouse model, cardiac grafts harvested from wild-type (WT) C57BL/6 mice were transplanted into the right neck of C57BL/6 LysM-GFP or CCR2 knockout $\left(\mathrm{CCR} 2^{-1-}\right.$ ) recipient mice after $1 \mathrm{~h}$ of cold $\left(4^{\circ} \mathrm{C}\right)$ ischemia as previously described (11). The ischemia-reperfusion injury mouse model was developed in 2- to 4-mo-old C57BL/6 mice by following a reported protocol (9).

\section{Radiolabeling of DOTA-ECL1i with ${ }^{64} \mathrm{Cu}$ and ${ }^{68} \mathrm{Ga}$}

The synthesis of DOTA-ECL1i and radiolabeling with ${ }^{64} \mathrm{Cu}$ and ${ }^{68} \mathrm{Ga}$ were reported previously $(9,12)$. The radiochemical purities for both tracers were more than $95 \%$ before administration to mice.

\section{PET/CT Imaging and Biodistribution}

All animal studies were performed in compliance with guidelines set forth by the National Institutes of Health Office of Laboratory Animal Welfare and approved by the Washington University institutional animal 
care and use committee. At 1-2 h after heart transplantation $(2,13)$, PET/CT (Siemens; 40- to 60-min dynamic scan) with ${ }^{64} \mathrm{Cu}$-DOTAECL1i was performed after tail-vein injection. For mice with ischemiareperfusion injury, PET/CT was done at day 3 after injury with ${ }^{68} \mathrm{Ga}$-DOTA-ECL1i and at day 4 with ${ }^{64} \mathrm{Cu}$-DOTA-ECL1i and quantified as previously reported $(9,12)$. A post-PET biodistribution study in the heart transplant model was performed immediately after imaging (8).

\section{Flow Cytometry}

To characterize the expression of CCR2, saline-perfused cardiac tissue specimens were collected and processed as previously reported. Fluorescence-activated cell sorting analysis was performed on an LSR II platform (BD) (9).

\section{Autoradiography}

Human pathologic specimens were obtained under Institutional Review Board protocol 201305086, approved by the Washington University Human Research Protection Office. Fixed sections of human heart tissue were used to test the binding of ${ }^{64} \mathrm{Cu}$-DOTAECL1i to human CCR2. Competitive receptor blocking studies were also performed to verify the binding specificity, using previously reported methods $(8,9)$.

\section{Statistics}

Data were analyzed using GraphPad Prism (version 6.07). Differences between groups were compared using paired parametric or unpaired nonparametric 2-tailed $t$ tests. A $P$ value of less than 0.05 was indicative of a statistically significant difference.

\section{RESULTS}

The sensitivity and specificity of ${ }^{64} \mathrm{Cu}$-DOTA-ECL1i to image $\mathrm{CCR} 2+$ cells were assessed in mouse syngeneic heterotopic heart transplant models. In this model, hearts from WT mice were harvested, placed on ice for $1 \mathrm{~h}$, and subsequently transplanted in the cervical position of either a WT recipient mouse or a CCR2 $2^{-1-}$ mouse. As shown in Figure 1A, besides rapid renal clearance as previous reported (12), PET/CT imaging revealed intensive signal in the donor heart but minimal tracer retention in the native heart in the WT $\rightarrow$ WT mouse heart transplantation model. In the WT $\rightarrow$ $\mathrm{CCR}^{-/-}$model (Fig. 1B), a similar profile was detected, with tracer uptake in the donor heart and marginal accumulation in the native heart. Quantitative uptake analysis showed that tracer accumulation in the donor heart $(3.51 \pm 0.19$ percentage injected dose $[\% \mathrm{ID}] / \mathrm{g}, n=4)$ was approximately 4 times higher than that in the native heart $(0.72 \pm 0.08 \% \mathrm{ID} / \mathrm{g}, P<0.0001, n=4)$ in the $\mathrm{WT} \rightarrow \mathrm{WT}$ model (Fig. 1C). In the WT $\rightarrow \mathrm{CCR} 2^{-1-}$ model (Fig. 1C), a 5-fold higher difference in tracer accumulation was determined in the donor heart $(1.46 \pm 0.16 \% \mathrm{ID} / \mathrm{g}, n=3)$ than in the native heart $(0.25 \pm 0.06 \% \mathrm{ID} / \mathrm{g}, P<0.0001, n=3)$. Moreover, the donor heart tracer uptake in the WT $\rightarrow$ WT model was 1.4-fold $(P<0.0001, n=3-4)$ higher than that in the $\mathrm{WT} \rightarrow \mathrm{CCR} 2^{-1-}$ model, whereas the difference was approximately 5-fold $(P<$ $0.0001, n=3-4)$ for the native hearts in the 2 models. This difference was likely a result of reduced infiltration of recipient $\mathrm{CCR} 2+$ cells from $\mathrm{CCR} 2^{-1-}$ recipients into the graft, as was reported in the ischemia-reperfusion injury model after lung transplantation (12). Of note, tracer uptake was evident within donor hearts transplanted into CCR2 $2^{-1-}$ recipients. This finding is likely due to the persistence of CCR2 + resident macrophages after transplantation, a population of cells that are activated after ischemiareperfusion injury and orchestrate infiltration of peripheral monocytes and neutrophils $(2,13)$.

We have previously demonstrated that recipient CCR2+ monocytes and macrophages accumulated within the transplanted heart after transplantation (2). In agreement, flow cytometry analysis of the $\mathrm{WT} \rightarrow \mathrm{WT}$ model revealed abundant CCR $2+$ monocytes and macrophages in the transplanted donor heart as compared with the native heart $2 \mathrm{~d}$ after heterotopic heart transplantation (Fig. 1D). Furthermore, post-PET biodistribution showed that tracer uptake in the donor heart $(2.82 \pm 0.40 \% \mathrm{ID} / \mathrm{g})$ was 4 times that in the native heart $(0.70 \pm 0.19 \% \mathrm{ID} / \mathrm{g}, P<0.0001, n=4)$ (Fig. $1 \mathrm{E})$, supporting PET data. In contrast to the bone marrow uptake

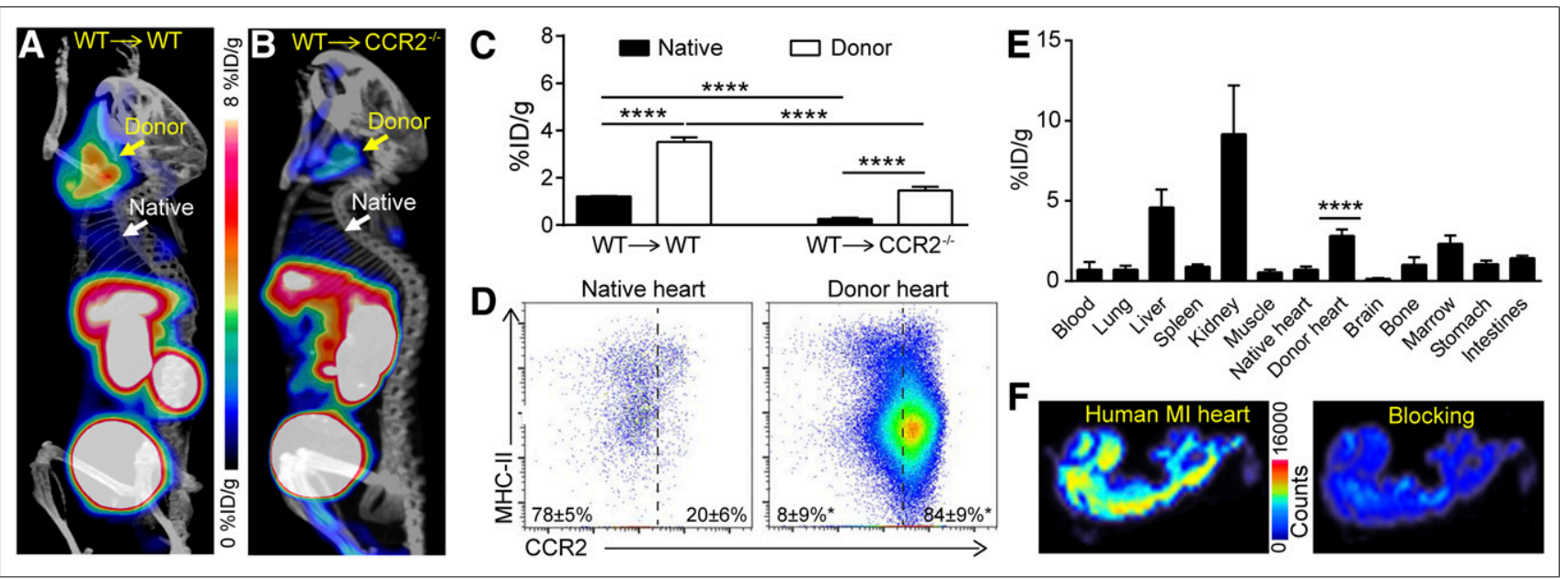

FIGURE 1. ${ }^{64} \mathrm{Cu}-\mathrm{DOTA}-\mathrm{ECL} 1 \mathrm{i}$ imaging of CCR2 in heart transplantation models. (A and B) ${ }^{64} \mathrm{Cu}-\mathrm{DOTA}-\mathrm{ECL} 1 \mathrm{i}$ PET/CT images (maximum-intensity projection) showing significant signal in transplanted heart (yellow arrow) and weak accumulation in native heart (white arrow) in WT $\rightarrow$ WT model (A) and WT $\rightarrow \mathrm{CCR} 2^{-/-}$model (B). (C) Quantitative uptake analysis of native and donor hearts of 2 models (WT $\rightarrow$ WT and WT $\rightarrow C C R 2^{-/-}$). (D) Flow cytometry assay showing 3-fold more CCR2 + cells in transplanted heart than in native heart in WT $\rightarrow$ WT model. (E) Post-PET biodistribution showing 3-fold higher uptake in donor heart than in native heart in WT $\rightarrow$ WT model. $(F)$ Autoradiographic images of ${ }^{64}$ Cu-DOTA-ECL1i on human MI specimens revealing heterogeneous tracer binding. Competitive blocking using excess nonradioactive ECL1i shows decreased tracer binding. ${ }^{\star * \star \star} P<0.0001$, assessed by 1-way ANOVA. 
$(0.57 \pm 0.16 \% \mathrm{ID} / \mathrm{g}, n=4)$ in naive mice $(8)$, tracer localization in the marrow $(2.33 \pm 0.51 \% \mathrm{ID} / \mathrm{g})$ of the $\mathrm{WT} \rightarrow \mathrm{WT}$ model was also 3 times higher, indicating the increased production and accumulation of $\mathrm{CCR} 2+$ monocytes within the bone marrow compartment.

To further assess the potential of ${ }^{64} \mathrm{Cu}$-DOTA-ECL1i for human cardiac injury imaging, the binding of ${ }^{64} \mathrm{Cu}$-DOTA-ECL1i to human CCR2 was evaluated in human MI specimens, which are known to contain CCR2 + monocytes and macrophages, using autoradiography (9). A substantial and heterogeneous binding profile was observed in human MI tissues, which was blocked by excess nonradioactive ECL1i peptide (Fig. 1F), indicating the specificity of ${ }^{64} \mathrm{Cu}$-DOTA-ECL1i binding to human hearts.

To further assess the suitability of ${ }^{64} \mathrm{Cu}$-DOTA-ECL1i for human cardiac injury imaging, we compared it with a ${ }^{68} \mathrm{Ga}$-radiolabeled analog in a previously established mouse MI model (9). ${ }^{68} \mathrm{Ga}$-DOTA-ECL1i and ${ }^{64} \mathrm{Cu}$-DOTA-ECL1i each showed significant radiotracer uptake within the infarct region 3-4 d after ischemiareperfusion injury (Figs. 2A and 2B). Quantitative analysis in the same mouse showed that the uptake of ${ }^{68} \mathrm{Ga}$-DOTA-ECL1i within the heart at day 3 after injury $(3.07 \pm 0.33 \% \mathrm{ID} / \mathrm{g}, n=6)$ was comparable to that acquired a day later using ${ }^{64} \mathrm{Cu}$-DOTA-ECL1i $(2.71 \pm 0.28 \% \mathrm{ID} / \mathrm{g}$, $n=6)$. These data are also comparable to ${ }^{68} \mathrm{Ga}$-DOTA-ECL1i uptake at day 4 after MI $(2.53 \pm 0.52 \% \mathrm{ID} / \mathrm{g}, n=6)(9)$, indicating equivalent sensitivity of ${ }^{64} \mathrm{Cu}$-DOTA-ECL1i and ${ }^{68} \mathrm{Ga}$-DOTA-ECL1i for detecting CCR2 + cells within the infarcted heart.

\section{DISCUSSION}

Clinically, the identification of reliable biomarkers of inflammation is required to facilitate a timely diagnosis of heart transplant rejection and primary graft dysfunction, to identify heart failure patients at increased risk of disease progression, to elucidate pathways that contribute to the pathogenesis of these entities, and to allow for the development and appropriate use of immunomodulatory therapies. It is known that CCR2 + macrophages generate damaging inflammation by recruiting additional monocytes and neutrophils that release cytokines and oxidative products. Removal of CCR2+ macrophages is sufficient to reduce chemokine and cytokine production and monocyte and neutrophil recruitment and results in preservation of left ventricular function and suppression of pathologic remodeling after MI and heart transplantation. Thus, the precise imaging of CCR2 + macrophages has the potential to determine the severity of heart injury, provide guidance for targeted treatment, and monitor treatment response.

Herein, we report robust detection of CCR $2+$ monocytes and macrophages in mouse models of heart injury using a clinical-grade ${ }^{64} \mathrm{Cu}$-DOTA-ECL1i PET tracer. The imaging sensitivity and specificity were demonstrated using $\mathrm{WT} \rightarrow \mathrm{WT}$ and $\mathrm{WT} \rightarrow \mathrm{CCR} 2^{-\prime-}$ heart transplantation models. We demonstrated that the ${ }^{64} \mathrm{Cu}$-DOTAECL1i tracer provides imaging efficacy equivalent to that of our previously published ${ }^{68} \mathrm{Ga}$-DOTA-ECL1i tracer in a mouse MI model. We further demonstrated that the ${ }^{64} \mathrm{Cu}$-DOTA-ECL1i tracer binds inflamed human myocardial tissue.

Compared with ${ }^{68} \mathrm{Ga}$, the radioactive decay half-life of ${ }^{64} \mathrm{Cu}$ is 10 times longer, providing an imaging agent that is ideal for multicenter translational studies. ${ }^{64} \mathrm{Cu}$-DOTA-ECL1i can be produced at a single center, and intact tracer can be shipped to collaborating institutions, avoiding the need for onsite ${ }^{68} \mathrm{Ge} /{ }^{68} \mathrm{Ga}$ generators or radiochemistry expertise necessary for local tracer production. More importantly, the ${ }^{64} \mathrm{Cu}$-DOTA-ECL1i tracer is currently approved by the U.S. Food and Drug Administration for clinical investigation under an exploratory investigational new drug application.

\section{CONCLUSION}

This study demonstrates the utility of ${ }^{64} \mathrm{Cu}$-DOTA-ECL1i to detect CCR2 + monocytes and macrophages in preclinical models and provides the requisite preclinical data to support translation of ${ }^{64} \mathrm{Cu}$-DOTA-ECL1i for clinical investigation of heart injuries.

\section{DISCLOSURE}

Yongjian Liu, Daniel Kreisel, Robert Gropler, Steven Brody, and Kory Lavine have a pending patent entitled "Compositions and Methods for Detecting CCR2 Receptors" (application 15/ 611,577). Daniel Kreisel serves on the Scientific Advisory Board of Compass Therapeutics. Yongjian Liu is supported by NIH R35 HL145212, R01 HL131908, and P41 EB025815. Kory Lavine is supported by NIH K08 HL123519, R01 HL138466, R01 HL139714, the Burroughs Welcome Fund (1014782), the Children's Discovery Institute of Washington University and St. Louis Children's Hospital (CHII-2015-462 and CH-II-2017-628), and the Foundation of Barnes-Jewish Hospital (8038-88). Daniel Kreisel is supported by 1P01AI116501, R01 HL094601, Veterans Administration Merit Review grant 1I01BX002730, and the Foundation for Barnes-Jewish Hospital. Steven Brody is supported by R01 HL131908 and is the Dorothy R. and Hubert C. Moog Professor of Pulmonary Medicine, awarded through the Barnes-Jewish Hospital Foundation. Robert Gropler is supported by P41 EB025815. No other potential conflict of interest relevant to this article was reported.

\section{ACKNOWLEDGMENTS}

FIGURE 2. ${ }^{64} \mathrm{Cu}-\mathrm{DOTA}-\mathrm{ECL} 1 \mathrm{i}$ compared with ${ }^{68} \mathrm{Ga}-\mathrm{DOTA}-\mathrm{ECL} 1 \mathrm{i}-$ mediated imaging of CCR2 in injured heart. Representative PET/CT images (transverse plane) of ${ }^{68} \mathrm{Ga}-\mathrm{DOTA}-\mathrm{ECL} 1 \mathrm{i}$ acquired at day $3(\mathrm{~A})$ and ${ }^{64} \mathrm{Cu}-\mathrm{DOTA}$-ECL1i acquired at day 4 (B) after $\mathrm{Ml}$ in same mouse show comparable PET signals $(C)$ in same infarcted region of heart.
We thank the small-animal imaging facility and cyclotron facility at Washington University for assistance with this research. 


\section{KEY POINTS}

QUESTION: What is the feasibility of noninvasively imaging proinflammatory CCR2+ cells after heart injury?

PERTINENT FINDINGS: ${ }^{64} \mathrm{Cu}-\mathrm{DOTA}-\mathrm{ECL} 1 \mathrm{i}$ showed sensitive and specific detection of CCR2 + cells in mouse heart injury models, as was further confirmed by flow cytometry analysis. The tracer also binds to injured human heart specimens.

IMPLICATIONS FOR PATIENT CARE: This study provides the requisite preclinical data to support translation of ${ }^{64} \mathrm{Cu}$-DOTAECL1i for clinical investigation of heart injuries and may provide new insight into the role of inflammation during progression of human heart failure.

\section{REFERENCES}

1. Bajpai G, Schneider C, Wong N, et al. The human heart contains distinct macrophage subsets with divergent origins and functions. Nat Med. 2018;24:12341245.

2. Bajpai G, Bredemeyer A, Li W, et al. Tissue resident CCR2- and CCR2+ cardiac macrophages differentially orchestrate monocyte recruitment and fate specification following myocardial injury. Circ Res. 2019;124:263-278.

3. Leuschner F, Dutta P, Gorbatov R, et al. Therapeutic siRNA silencing in inflammatory monocytes in mice. Nat Biotechnol. 2011;29:1005-1010.
4. Varasteh Z, Mohanta S, Robu S, et al. Molecular imaging of fibroblast activity after myocardial infarction using a ${ }^{68} \mathrm{Ga}$-labeled fibroblast activation protein inhibitor, FAPI-04. J Nucl Med. 2019;60:1743-1749.

5. Thackeray JT, Derlin T, Haghikia A, et al. Molecular imaging of the chemokine receptor CXCR4 after acute myocardial infarction. JACC Cardiovasc Imaging. 2015;8:1417-1426.

6. Bascunana P, Hess A, Borchert T, et al. ${ }^{11} \mathrm{C}$-methionine PET identifies astroglia involvement in heart-brain inflammation networking after acute myocardial infarction. J Nucl Med. December 5, 2019 [Epub ahead of print].

7. Li W, Luehmann HP, Hsiao HM, et al. Visualization of monocytic cells in regressing atherosclerotic plaques by intravital 2-photon and positron emission tomographybased imaging-brief report. Arterioscler Thromb Vasc Biol. 2018;38:1030-1036.

8. Liu Y, Gunsten SP, Sultan DH, et al. PET-based imaging of chemokine receptor 2 in experimental and disease-related lung inflammation. Radiology. 2017;283:758768.

9. Heo GS, Kopecky B, Sultan D, et al. Molecular imaging visualizes recruitment of inflammatory monocytes and macrophages to the injured heart. Circ Res. 2019;124: 881-890.

10. PET imaging CCR2 in lung inflammation. ClinicalTrials.gov website https:// clinicaltrials.gov/ct2/show/NCT03492762?term $=$ CCR2 + PET\&draw $=2 \&$ rank $=2$. Published April 10, 2018. Updated May 7, 2020. Accessed June 30, 2020.

11. Li W, Nava RG, Bribriesco AC, et al. Intravital 2-photon imaging of leukocyte trafficking in beating heart. J Clin Invest. 2012;122:2499-2508.

12. Liu Y, Li W, Luehmann HP, et al. Noninvasive imaging of CCR2(+) cells in ischemia-reperfusion injury after lung transplantation. Am J Transplant. 2016;16:30163023.

13. Li W, Hsiao HM, Higashikubo R, et al. Heart-resident CCR2(+) macrophages promote neutrophil extravasation through TLR9/MyD88/CXCL5 signaling. JCI Insight. 2016;1:e87315. 